\title{
Polymer Measure: Varadhan's Renormalization Revisited
}

\author{
Wolfgang Bock \\ CMAF, University of Lisbon, P 1649-003 Lisbon, Portugal \\ bock@campus.ul.pt \\ Maria João Oliveira \\ Universidade Aberta, P 1269-001 Lisbon, Portugal \\ CMAF, University of Lisbon, P 1649-003 Lisbon, Portugal \\ oliveira@cii.fc.ul.pt \\ José Luís da Silva \\ CCEE, University of Madeira, P 9000-390 Funchal, Portugal \\ CCM, University of Madeira, P 9000-390 Funchal, Portugal \\ luis@uma.pt \\ Ludwig Streit \\ Forschungszentrum BiBoS, Bielefeld University, D 33501 Bielefeld, Germany \\ CCM, University of Madeira, P 9000-390 Funchal, Portugal \\ streit@physik.uni-bielefeld.de
}

\begin{abstract}
Through chaos decomposition we improve the Varadhan estimate for the rate of convergence of the centered approximate self-intersection local time of planar Brownian motion.
\end{abstract}

Keywords: Edwards model, self-intersection local time, Varadhan renormalization, white noise analysis

Mathematics Subject Classifications (2010): 28C20, 41A25, 60H40, 60J55, 60J65, 82D60 


\section{Introduction}

The Edwards model [2] for self-repelling or "weakly self-avoiding" $d$-dimensional Brownian motion, with applications in polymer physics and quantum field theory, is informally given by a Gibbs factor

$$
G=\frac{1}{Z} \exp \left(-g \int_{0}^{T} d s \int_{0}^{t} d t \delta(B(s)-B(t))\right)
$$

with $g>0$ and

$$
Z=E\left(\exp \left(-g \int_{0}^{T} d s \int_{0}^{t} d t \delta(B(s)-B(t))\right)\right)
$$

Using

$$
\delta_{\varepsilon}(x):=\frac{1}{(2 \pi \varepsilon)^{d / 2}} e^{-\frac{|x|^{2}}{2 \varepsilon}}, \quad \varepsilon>0,
$$

one defines an approximate self-intersection local time by

$$
L_{\varepsilon}:=\int_{0}^{T} d t \int_{0}^{t} d s \delta_{\varepsilon}(B(t)-B(s)) .
$$

For $d \geq 2$

$$
\lim _{\varepsilon \searrow 0} \mathbb{E}\left(L_{\varepsilon}\right)=\infty
$$

For the planar case $d=2$, Varadhan [3] has shown that centering

$$
L_{\varepsilon, c}:=L_{\varepsilon}-\mathbb{E}\left(L_{\varepsilon}\right) \text { and } L_{c}:=\lim _{\varepsilon \searrow 0} L_{\varepsilon, c}
$$

is sufficient to make the Gibbs factor $G=Z^{-1} \exp \left(-g L_{c}\right)$ well defined. An estimate for the rate of convergence

$$
\left\|L_{c}-L_{\varepsilon, c}\right\|_{2}^{2} \leq \text { const. } \varepsilon^{\alpha}
$$

for all $\alpha<1 / 2$, is in Varadhan's words, "the most difficult step of all and requires considerable estimation". In this note we shall use a multiple Wiener integral or chaos expansion for an alternate and comparatively straightforward argument, extending the estimate to all $\alpha<1$. 


\section{Fock space representation of the local time}

The Ito-Segal-Wiener isomorphism relates the $L^{2}$ space of planar Brownian motion with the Fock space

$$
\mathfrak{F}=\left(\bigoplus_{n=0}^{\infty} \operatorname{Sym} L^{2}\left(\mathbb{R}^{n}, n ! d^{n} x\right)\right)^{\otimes 2}
$$

We shall use the multi-index notation

$$
\mathbf{n}=\left(n_{1}, n_{2}\right) \quad n=n_{1}+n_{2}, \quad \mathbf{n} !=n_{1} ! n_{2} !
$$

The Fock space norm is then

$$
\|F\|_{2}^{2}=\sum_{n_{i} \geq 0} \mathbf{n} !\left\|F_{\mathbf{n}}\right\|_{2}^{2}
$$

For $L_{\varepsilon, c}$ the kernel functions $F_{\mathbf{n}}$ were computed explicitly in [1]. For the planar case the result is

Proposition 1 [1]: For $d=2$ the kernel functions $F_{\mathbf{n}}$ of $L_{\varepsilon, c}(T)$ and $L_{c}(T)$ have their support on $[0, T]^{n}$ and are, with $\varepsilon>0$, and $\varepsilon=0$ respectively, for $n>1$

$$
\begin{aligned}
& F_{2 \mathbf{n}, \varepsilon}\left(u_{1}, \ldots, u_{2 n}\right)=\frac{1}{2 \pi}\left(-\frac{1}{2}\right)^{n} \frac{1}{n(n-1) \mathbf{n} !} \\
& \times\left(\frac{1}{(T+\varepsilon)^{n-1}}-\frac{1}{(v+\varepsilon)^{n-1}}-\frac{1}{(T-u+\varepsilon)^{n-1}}+\frac{1}{(v-u+\varepsilon)^{n-1}}\right),
\end{aligned}
$$

where $v:=\max _{1 \leq k \leq 2 n} u_{k} \leq T$ and $u:=\min _{1 \leq k \leq 2 n} u_{k} \geq 0$. For $n=1$

$$
F_{2, \varepsilon}\left(u_{1}, u_{2}\right)=-\frac{1}{4 \pi}(\ln (v+\varepsilon)+\ln (T-u+\varepsilon)-\ln (v-u+\varepsilon)-\ln (T+\varepsilon)) .
$$

All kernel functions $F_{\mathbf{n}}$ with odd $n_{i}$ are zero.

\subsection{The rate of convergence}

Theorem 2 Given $T>0$. Then for any $\alpha<1$ there is a constant $C_{T, \alpha}>0$ such that for all $\varepsilon>0$

$$
\left\|L_{\varepsilon, c}(T)-L_{c}(T)\right\|_{2}^{2} \leq C_{T, \alpha} \varepsilon^{\alpha} .
$$


Proof: From Proposition 1

$$
\left\|F_{2 \mathbf{n}, 0}-F_{2 \mathbf{n}, \varepsilon}\right\|_{2}^{2}=\left(n(n-1) 2 \pi 2^{n} \mathbf{n} !\right)^{-2} \int_{0}^{T} d^{2 n} u_{k} K_{\varepsilon}^{2}(u, v, T)
$$

where for $n>1$

$$
\begin{aligned}
K_{\varepsilon}(u, v, T)= & \left(T^{-n+1}-(T+\varepsilon)^{-n+1}\right)-\left(v^{-n+1}-(v+\varepsilon)^{-n+1}\right) \\
& -\left((T-u)^{-n+1}-(T-u+\varepsilon)^{-n+1}\right)+\left((v-u)^{-n+1}-(v-u+\varepsilon)^{-n+1}\right) .
\end{aligned}
$$

Since $K_{\varepsilon}(u, v, T)$ does not depend on $2 n-2$ of the $u_{k}$-variables, we may integrate them out:

$$
\left\|F_{2 \mathbf{n}, 0}-F_{2 \mathbf{n}, \varepsilon}\right\|_{2}^{2}=\left(n(n-1) 2 \pi 2^{n} \mathbf{n} !\right)^{-2} 2 n(2 n-1) \int_{0}^{T} d v \int_{0}^{v} d u(v-u)^{2 n-2} K_{\varepsilon}^{2}(u, v, T) .
$$

Of the four terms in $K_{\varepsilon}$, the last one is dominant so that

$$
\begin{aligned}
\left\|F_{2 \mathbf{n}, 0}-F_{2 \mathbf{n}, \varepsilon}\right\|_{2}^{2} & \leq 16 \frac{2 n(2 n-1)}{\left(n(n-1) 2 \pi 2^{n} \mathbf{n} !\right)^{2}} \int_{0}^{T} d v \int_{0}^{v} d u\left(1-\left(\frac{v-u}{v-u+\varepsilon}\right)^{n-1}\right)^{2} \\
& =16 \frac{2 n(2 n-1)}{\left(n(n-1) 2 \pi 2^{n} \mathbf{n} !\right)^{2}} \int_{0}^{T} d \tau \int_{0}^{T-\tau} d u\left(1-\left(\frac{\tau}{\tau+\varepsilon}\right)^{n-1}\right)^{2} \\
& \leq 16 \frac{2 n(2 n-1) T}{\left(n(n-1) 2 \pi 2^{n} \mathbf{n} !\right)^{2}} \int_{0}^{T} d \tau\left(1-\left(\frac{\tau}{\tau+\varepsilon}\right)^{n-1}\right)^{2} \\
& =16 \frac{2 n(2 n-1) T}{\left(n(n-1) 2 \pi 2^{n} \mathbf{n} !\right)^{2}} \int_{0}^{T} d \tau\left(\tau^{n-1}(n-1) \int_{0}^{\varepsilon} \frac{d x}{(x+\tau)^{n}}\right)^{2}(1)
\end{aligned}
$$

By Hölder's inequality

$$
\int_{0}^{\varepsilon} \frac{d x}{(x+\tau)^{n}} \leq \varepsilon^{1 / q}\left(\int_{0}^{\infty} \frac{d x}{(x+\tau)^{n p}}\right)^{1 / p}=\varepsilon^{1 / q}\left(\frac{1}{n p-1} \tau^{1-n p}\right)^{1 / p}
$$

if $\frac{1}{q}+\frac{1}{p}=1$. Insertion of this estimate into (1) produces

$$
\sum_{\mathbf{n}: n>1}(2 \mathbf{n}) !\left\|F_{2 \mathbf{n}, 0}-F_{2 \mathbf{n}, \varepsilon}\right\|_{2}^{2} \leq \frac{4 p}{(2-p) \pi^{2}} T^{\frac{2}{p}} \varepsilon^{\frac{2}{q}} \sum_{\mathbf{n}: n>1}(2 \mathbf{n}) ! \frac{2 n(2 n-1)}{\left(n 2^{n} \mathbf{n} !\right)^{2}} \frac{1}{(p n-1)^{\frac{2}{p}}}
$$


which is convergent if $\frac{2}{p}>1$, i.e., $q>2$. For the $n=1$ term an $\varepsilon^{\frac{2}{q}}$ estimate is likewise obtained via Hölder's inequality.

Hence, for any $\alpha<1$,

$$
\left\|L_{c}(T)-L_{\varepsilon, c}(T)\right\|_{2}^{2}=\sum_{\mathbf{n}: n \geq 1}(2 \mathbf{n}) !\left\|F_{2 \mathbf{n}, 0}-F_{2 \mathbf{n}, \varepsilon}\right\|_{2}^{2} \leq C(T, \alpha) \varepsilon^{\alpha}, \quad \forall \varepsilon>0 .
$$

\section{Acknowledgments}

Financial support of FCT through the research project PTDC/MAT-STA/1284/2012 is gratefully acknowledged. W. B. and J. L. S. also would like to thank for financial support of IGK and DFG through SFB-701 (University of Bielefeld), respectively.

\section{References}

[1] M. de Faria, T. Hida, L. Streit and H. Watanabe: Intersection local times as generalized white noise functionals, Acta Appl. Math. 46, 351362 (1997).

[2] S. F. Edwards: The statistical mechanics of polymers with excluded volume. Proc. Roy. Soc. 85, 613-624 (1965).

[3] S. R. S. Varadhan: Appendix to "Euclidian quantum field theory" by K. Szymanzik, in: R. Jost ed., Local Quantum Theory. Academic Press, New York, 1969. 